Accepted for publication in International Journal of Polymeric Materials and Polymeric Biomaterials

Published in 2019

DOI: $10.1080 / 00914037.2018 .1563083$ 


\title{
Electrospun Fiber Mats from Poly(alloocimene-isobutylene-alloocimene) Thermoplastic Elastomer
}

\author{
Aditya Jindal ${ }^{1}$, Kolos Molnár ${ }^{2,3}$, Andrew McClain ${ }^{1}$, Bruno Paiva dos Santos ${ }^{4}$, Melissa \\ Camassola ${ }^{4}$, and Judit E. Puskas ${ }^{1, *}$
}

\begin{abstract}
${ }^{1}$ The University of Akron, Department of Chemical and Biomolecular Engineering, Akron, $\mathrm{OH}$ 44325, USA

${ }^{2}$ Department of Polymer Engineering, Faculty of Mechanical Engineering, Budapest University of Technology and Economics, Müegyetem rkp. 3., H-1111, Budapest, Hungary

${ }^{3}$ MTA-BME Research Group for Composite Science and Technology, Mủegyetem rkp. 3., H1111, Budapest, Hungary

${ }^{4}$ Programa de Pós-Graduação em Biologia Celular e Molecular Aplicada à Saúde, University Luterana of Brasil (ULBRA), Canoas, Rio Grande do Sul 92425, Brazil.
\end{abstract}

*Corresponding author: Judit E. Puskas; jpuskas@ uakron.edu; 330-972-6203.

\begin{abstract}
A new polyisobutylene-based thermoplastic elastomer, poly(alloocimene-b-isobutylene-ballooci-mene triblock copolymer was synthesized by carbocationic polymerization, then electrospun intofree-standing rubbery fiber mats by adding $20 \mathrm{wt} \%$. poly(ethylene glycol) to the solution. AlthoughAIBA is a highly hydrophobic material that is non-polar and therefore has high electric resistivity,fiber mats were still successfully obtained. We found that poly(ethylene glycol) was fully embeddedinto the electrospun fibers. The tensile strength measured on microdumbbells was $2.7 \mathrm{MPa}$ at $537 \%$ elongation that is comparable to that of soft human tissues. These rubbery fiber mats were foundto be hydrophobic and cell culture studies showed their non-cytotoxicity. Based on these favorableproperties, these fiber mats show a great promise for tissue scaffold and drug delivery applications.
\end{abstract}


Keywords: thermoplastic elastomer; electrospinning; cytotoxicity; electron microscopy; XPS; mechanical properties.

\section{INTRODUCTION}

Electrospinning is a versatile and unique technique to producefibers in the range of microns to nanometers from polymer solutions using electrostatic forces [1]. These fibers with smaller pores and higher surface area have great potential in the biomedical industry because of the ease of fabrication of fibers from a wide array of polymers - biodegradable, non-degradable, synthetic, natural, or their blends. Some synthetic biocompatible polymers can be easily spun into fiber mats to make stretchable wound dressings, flexible scaffolds for cell growth and tissue engineering, and implantable membranes/coatings with the capability of controlled drug delivery.

Elastomers, which have the elasticity of natural rubber, are widely used in industry due to their durable and tough nature. Since elastomers have low glass transition temperature, it is very difficult to electrospin them into stable nanofibers. Also, the electrospun fibers on the collector may fuse quickly into large fibers or sometimes even a continuous film [2]. Electrospinning of thermoplastic elastomers (TPEs) is easier since they can be processed as plastics but exhibit elastomeric properties. Various TPEs have been electrospun in the last two decades. Sencadas et al. studied the electrospinning of poly(styrene-b-butadiene-b-styrene) (SBS) TPE into fiber membranes and showed that the membranes were hydrophobic with a contact angle of 132 and the tensile strength was $0.525 \mathrm{MPa}$ with 345\% elongation at break [3].

These authors also explained that the mechanical strength of the mat was lower than that of the bulk material (SBS) because of the higher porosity of the fibrous membrane. Since the fibers were 
not orderly arranged in the nonwoven membrane, only a small portion of the fibers resisted to the applied mechanical loading which caused less chain entanglements per unit mass of the porous membrane. The electrospinning of poly(styrene-b-isoprene-b-styrene) TPEs was demonstrated by Supaphol et al. and they produced fibers in the range of 2.7-16 $\mathrm{mm}$ [4]. Although electrospinning can produce ultrafine fibers, their fibers were uncharacteristically large. They theorized that TPE molecules usually stretch while flowing through a restricted channel of the nozzle and after leaving recoiling occurs very fast which could prevent Coulombic stretching to decrease the fiber diameter. Similarly, larger fiber diameter $(6 \mathrm{~mm})$ was also found for poly(styrene-b-(ethylene-co-1-butene)b-styrene) triblock copolymer (SEBS) fibers[5]. Our research group has been focusing on polyisobutylene (PIB)-based TPEs because of the excellent bioinertness and biostability of PIB. A linear poly(styrene-b-isobutylene-bstyrene) triblock copolymer (L_SIBS) TPE is used in clinical practice as a polymer matrix of a drug-eluting coating on the TAXUSTM coronary stent[6]. Over 6 million patients have benefited from this device, emphasizing the significance of PIB-based biomaterials. Due to the unique low permeability of L_SIBS, sustained drug delivery is achieved, but only approximately $10 \%$ of the encapsulated drug, Taxol, elutes from the coating, which is therapeutic for this application. After the success of L_SIBS, we developed another TPE, Arbomatrix, comprising a branched (arborescent or dendritic) PIB core and end blocks of poly(pmethylstyrene) (PMS)[7,8]. Arbomatrix was also shown to be bioinert in a rabbit model[9]. ElectroNanosprayTM, a technology of generating high velocity spray of nanoparticles, was used to coat several batches of Arbomatrix polymers loaded with Dexamethasone (DXM), a model drug, onto coronary stents. This particulate coating did not have an initial burst release but exhibited slow continuous release over time (20-40\% release in 28 days)[10]. For biomedical applications requiring more release, we theorized that encapsulating drugs into electrospun fiber 
mats would provide high surface area to volume ratio to release more drug. However, Liu et al.[11] reported that neat L_SIBS could not be electrospun, attributing this to the non-conductivity of the polymer solution. We reported conditions under which we electrospun neat L_SIBS and Arbomatrix onto aluminum stubs[12]. Subsequently we developed a new method that produced self-supporting fiber mats by electrospinning from a mixture of Arbomatrix and low molecular weight poly(ethylene glycol), PEG. The ratio of Arbomatrix to PEG was chosen to be 80/20 wt/wt based on scouting experiments. We successfully encapsulated a model drug, Zafirlukast, into the fibers, and demonstrated greater than $90 \%$ release[13]. Although electrospinning can produce ultrafine fibers, the mean fiber size for Zafirlukast-loaded Arbomatrix/PEG fibers was larger (4.197 $( \pm 0.580) \mathrm{mm})$. However, this system showed higher doses and slower release rates than a recent study using poly(lactic-co-glycolic acid) polymer coating with a similar drug for reducing the capsular contracture (an inflammatory response around silicone rubber breast prostheses) invivo[14]. Against this background, we report in this paper the electrospinning of a new linear PIB-based TPE, poly(alloocimene-b-isobutylene-b-alloocimene) or AIBA for short for which the chemical structure is shown in Figure S1. It is also a triblock copolymer like L_SIBS but contains polyalloocimene hard blocks instead of polystyrene. It is synthesized by the carbocationic copolymerization of isobutylene (IB) with alloocimene (Allo)[15]. Allo is produced by the thermal isomerization of a-pinene[16], a naturally occurring cyclic terpene found in the oils of several tree species. AIBA is easier to synthesize than Arbomatrix[17] and it has higher tensile strength (15 MPa and 600\% elongation at break) than Arbomatrix (5.6 MPa and 290\% elongation at break).

Electrospinning of non-polar and highly non-conductive materials such as AIBA is a challenge, because the process employs high voltage to electrically charge the polymer jets to produce ultrafine fibers[1]. Therefore, the polymer was mixed with PEG to enhance the electrical 
conductivity in order to produce self-supporting fiber mats. AIBA is a candidate for implanted drug delivery systems. The AIBA electrospun fiber mats were therefore undergone cell culture and tensile studies to investigate their bioinertness and biofunctionality.

\section{EXPERIMENTAL}

\subsection{Materials}

AIBA (PAllo = $\left.32.34 \mathrm{wt} \%, \mathrm{M}_{\mathrm{w}}=544,260 \mathrm{~g} / \mathrm{mol}, \mathrm{M}_{\mathrm{w}} / \mathrm{M}_{\mathrm{n}}=1.41\right)$ was synthesized as reported [15]. Poly(ethylene glycol) PEG $\left(\mathrm{M}_{\mathrm{n}}=2000 \mathrm{~g} / \mathrm{mol}\right)$ was purchased from Sigma-Aldrich (USA) and was used as received. Tetrahydrofuran (THF, Fisher, USA) and toluene (reagent grade, Aldrich, USA) were used without further purification. Complete culture medium (CCM) was composed of Dulbecco's modified Eagle's medium supplemented with $10 \%$ fetal bovine serum (Cultilab, São Paulo, Brazil) for fibroblasts. $\mathrm{Ca}^{2+}$ and $\mathrm{Mg}^{2+}$ free Hank's balanced salt solution was used for washing tissues and cells. All reagents were from Sigma Chemical (St. Louis, MO). NCTC clone 929 (strain L), known as L929 mouse fibroblasts, were obtained from Banco de Células do Rio de Janeiro (Rio de Janeiro, Brazil).

\subsection{Electrospinning}

AIBA/PEG in a mass ratio of 80/20 was dissolved in 95/5 THF/toluene (wt/wt) solvent mixture to make a concentration of $12.5 \mathrm{wt} \%$ for electrospinning. Typical conditions used were: $12 \mathrm{kV}, 30$ gauge needle (inner diameter $=160 \mu \mathrm{m})$, feed volume rate of $40 \mu \mathrm{L} / \mathrm{min}, 20 \mathrm{~cm}$ distance from the 
collector, room temperature, and $32 \%$ relative humidity. A direct current high voltage supply was purchased from Gamma High Voltage Research, Ormond Beach, Florida, USA, and the solution was fed through a syringe pump (New Era Pump Systems, Inc., USA).

\subsection{Fiber Mat Characterization}

\subsubsection{Scanning Electron Microscopy (SEM)}

Morphology was investigated by a JEOL JSM-7401F instrument. The mean fiber size was measured using the FibraQuant software (Nanoscaffold Technologies, LLC) from 5 images. The results are presented as mean \pm standard deviation (S.D.) of 200 individual measurements.

\subsubsection{X-ray Photoelectron Spectroscopy (XPS)}

XPS analysis was conducted by PHI Versa Probe II Scanning XPS Microprobe using three scans taken over $100 \mu \mathrm{m}$ beam diameter at $45^{\circ}$ incidence angle.

\subsubsection{Water Contact Angle (WCA) Measurements}

A goniometer (Ramé-Hart Instrument Co., Netcong, NJ) was used for WCA imaging ( 5 x $5 \mu \mathrm{L}$ water droplets for each sample). For the static WCA, the image was taken at 5 seconds, while for advancing and receding WCA, the images were taken as soon as the droplet contact area expanded or shrunk on the surface (within 15 seconds). The ImageJ software (National Institutes of Health) was used to process the data. The results are presented as mean \pm standard deviation from five measurements.

\subsubsection{Tensile Tests}


The quasi-static tensile measurements were carried out on an Instron 5567 instrument, equipped with a $1 \mathrm{kN}$ load cell using S3A type (DIN 53504) microdumbbell specimens (total length $=45 \mathrm{~mm}$, neck length $=25 \mathrm{~mm}$, and neck width $=3 \mathrm{~mm}$ ), a cross-head speed of $500 \mathrm{~mm} / \mathrm{min}$ according to ASTM 412-06a. The grip distance was $40 \mathrm{~mm}$. The tensile stress was calculated using the initial thickness of the dumbbell specimens $(n=3)$.

\subsection{Cell Culture Studies}

Cytotoxicity assays were performed according to ISO standards [17]. Mouse fibroblast L929 cells were seeded onto a tissue culture plate with 96 wells $\left(10^{4}\right.$ cells/well; medium volume: $\left.100 \mu \mathrm{L}\right)$ and incubated in direct contact with the fiber mats for 72 hours. A second set had fiber mats incubated in $200 \mu \mathrm{L}$ of culture medium $/ \mathrm{cm}^{2}$ fiber mats for 24 hours. The culture medium was removed and used to culture cells for 72 hours $\left(10^{4}\right.$ cells/well; medium volume: $\left.100 \mu \mathrm{L}\right)$. Cells were observed every day using light microscopy (Axiovert 25; Zeiss, Hallbergmoos, Germany) and photos were taken using a digital camera (AxioCam MRc, Zeiss) and the software AxioVision 3.1- Zeiss. After 72 hours the medium was removed and $50 \mu \mathrm{L}$ of 3-(4,5-Dimethylthiazol-2-yl)-2,5Diphenyltetrazolium Bromide (MTT) $(1 \mathrm{mg} / \mathrm{mL})$ and $100 \mu \mathrm{L}$ DMSO was added. UV absorbance at $\lambda=570 \mathrm{~nm}$ was measured. All sets were run in triplicate. The optical density was converted to cellular activity as percentage to negative control. Analysis of variance (ANOVA) for single and multiple factor comparisons was conducted.

\section{RESULTS AND DISCUSSION}

\subsection{Fiber Morphology}


The AIBA batch used in this paper was synthesized similarly to that reported in reference[15]. The 1H NMR spectrum in Figure S2 shows that the PAllo blocks have mostly 4-7 enchainment with a small amount of conjugated diene in 2-7 enchainment, similarly to that reported earlier[15]. DSC of AIBA showed two Tgs at _65 _ $\mathrm{C}$ and $70 \_\mathrm{C}$ for the PIB and PAllo segments, respectively[15]. We tried DSC tests on the fiber mats, but because of the large free volume we did not get visible transitions. Figure 1 shows a representative SEM image of the electrospun fiber mat. The fibers assumed a dumbbell-shaped cross-section, as was reported earlier for Arbomatrix[12,13]. Reneker et al.[18] explained that the dumbbell-shape can form when the diametrically opposite parts of the polymer skin forming during electrospinning come into contact, yielding two small parallel tubes connected by a "bar". Figure S3 shows an image of the selfsupporting fiber mat and an enlarged image of fibers with a dumbbell crosssection. The mean fiber size (ribbon width) was found to be $4.12( \pm 0.83) \mathrm{mm}$ (nL209). XPS detected only carbon signals and traces of oxygen on the surface of the fibers (Figure S4 in Supporting Information). This agreed with earlier findings [13] therefore we concluded that the PEG was fully embedded into the fibers. Soaking in water for 2 days extracted the PEG from the AIBA/PEG fibers, similarly to that shown for Arbomatrix/PEG[4]. The XPS spectra before and after soaking are shown in Figures S4 and S5. Before soaking, only traces of oxygen were detected, indicating that very little if any PEG was present on the surface. After soaking, 12.3 atomic \% oxygen was detected, indicating that PEG migrated to the surface under water. Water contact angle (WCA) measurements showed high hydrophobicity and surface rearrangement. The static WCA was $121_{-} \pm 3_{-}$, while the dynamic WCA were $133_{-} \pm 2 \_$(advancing) and $27 \_ \pm 5 \_$(receding), signifying the segregation of the polar PEG to the surface (Figure S6). Comparable results were shown for Arbomatrix/PEG 
fibers previously[13]. It was shown for PIB-based TPEs that surface rearrangement could take place in an aqueous environment[19], due to chain mobility of the rubbery matrix.

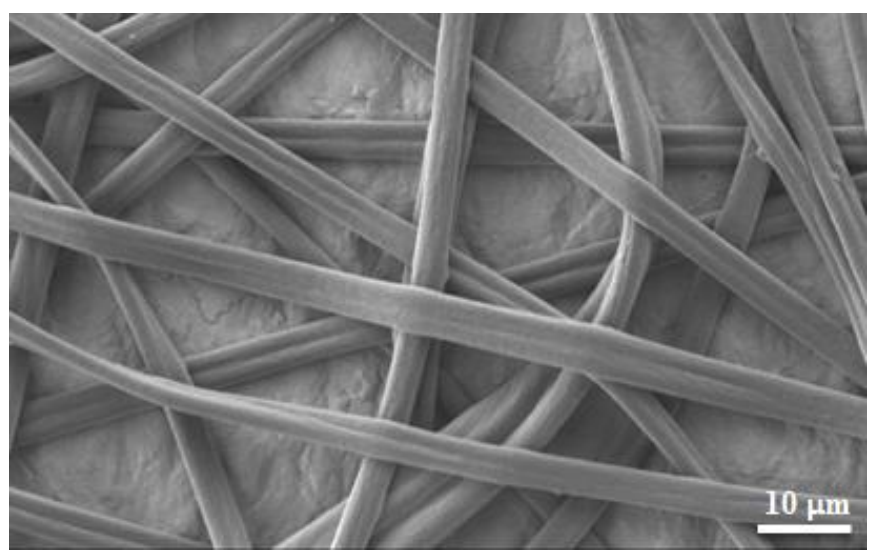

Figure 1. SEM image of electrospun fiber mat made from AIBA/PEG 80/20 in 95/5 THF/toluene.

\subsection{Tensile Tests}

Figure 2 shows a typical stress-strain plot of the electrospun fiber mats and Table 1 lists the data.

Table 1. Tensile properties of fiber mat microdumbbells

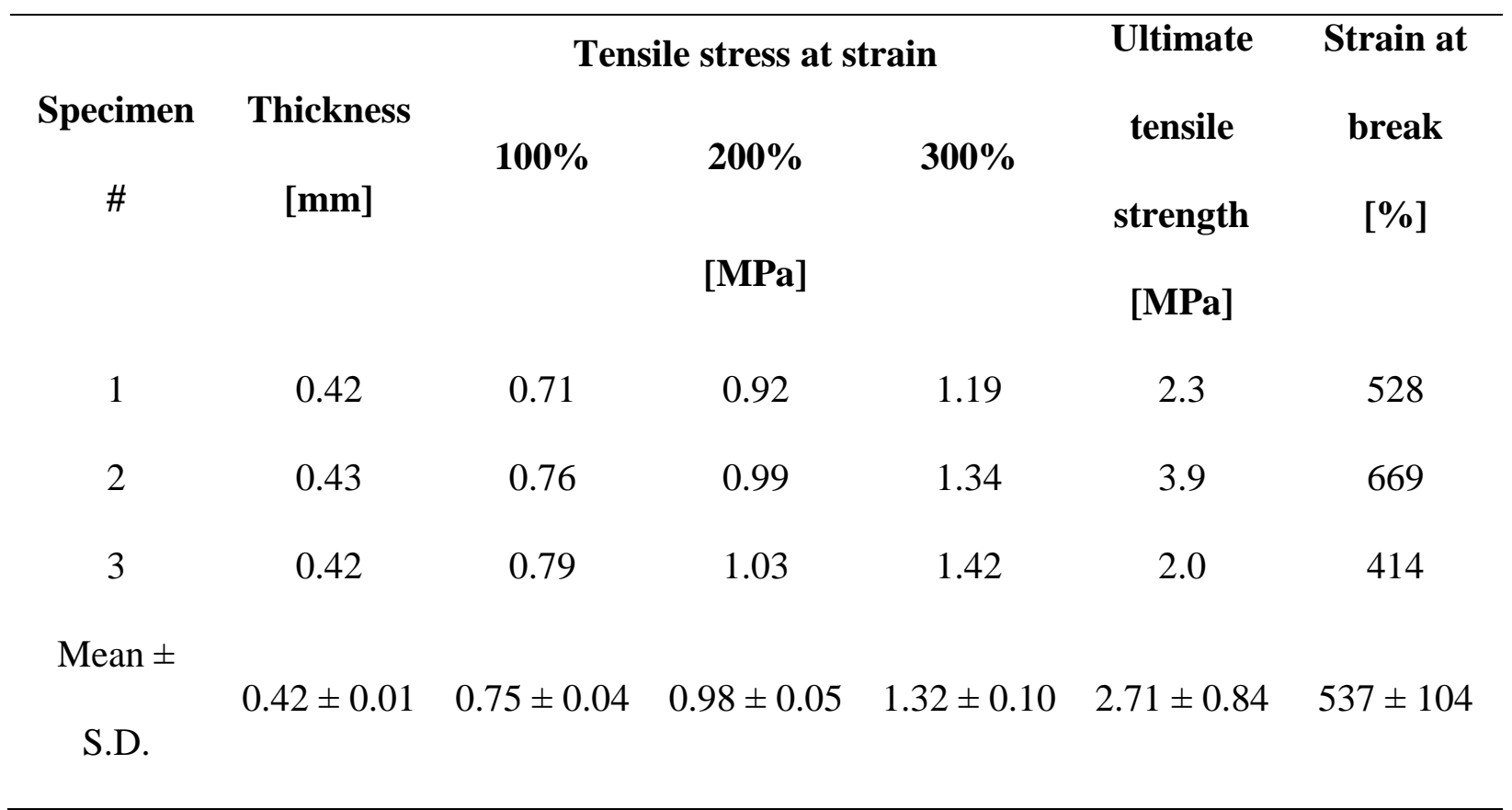




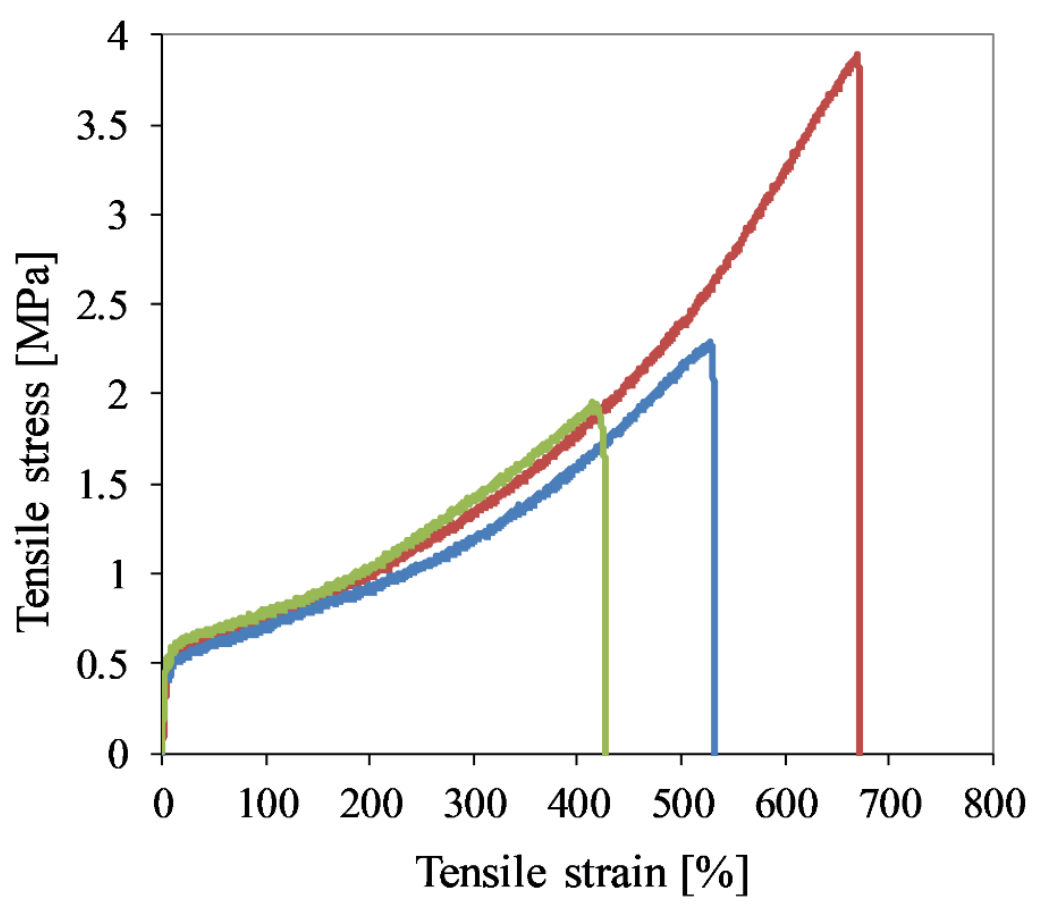

Figure 2. Tensile stress-strain plot of an electrospun fiber mats.

The plot represents TPE behavior with a rubbery plateau, and strain hardening - typical of PIBbased TPEs undergoing strain-induced crystallization. The plot represents TPE behavior with a rubbery plateau, and strain hardening - typical of PIB-based TPEs undergoing strain-induced crystallization. The tensile strength and elongation values in Table 1 are higher than those of soft tissues (0.3-3 MPa and 50-100\% elongation[20]) and so these fiber mats could be used as implants to withstand mechanical load and related deformations inside the human body. One of the implant applications is to reduce the inflammation around silicone rubber breast prostheses after mastectomy by wrapping those with the drug-eluting fiber mat.

\subsection{Cytotoxicity Studies}


Figure 3 shows that the AIBA/PEG fiber mats did not adversely affect cell viability after 72 hours incubation time, either in direct contact with the cells or after extraction. The horizontal line in Figure 3 marks the $70 \%$ cell viability threshold over which materials are considered non-cytotoxic [17]. The DMSO positive controls are below the line, indicating cytotoxicity. Cell viability was identical in the presence and in the absence of the mat. This is consistent with the bioinertness shown for PIB-based TPEs [13].

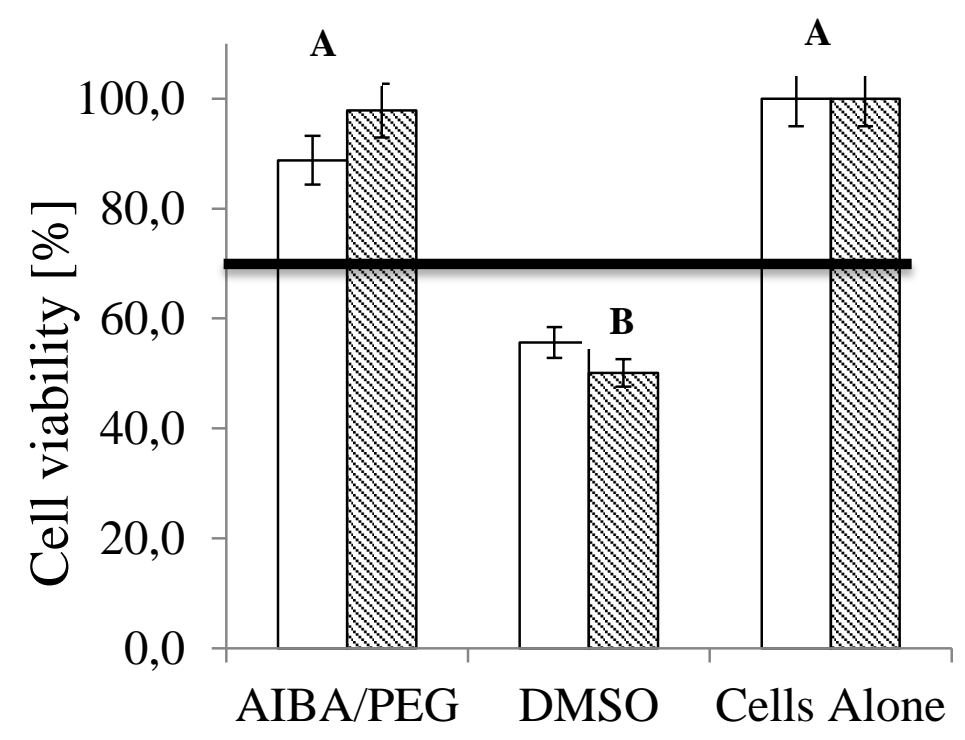

Figure 3. Bar graph of cell viability in direct contact (white bar) and in elution media (shaded bar); horizontal line at 70\% indicates acceptable threshold [17]. Positive control: DMSO; $\mathrm{n}=3$. Groups with different letters are significantly different, based on ANOVA with post hoc analysis $(\alpha=0.05)$. The error bars represent \pm standard deviation.

\section{CONCLUSIONS}

Self-standing fiber mats were successfully electrospun from AIBA/PEG. The mean fiber size was $4.12( \pm 0.87) \mathrm{mm}$. XPS showed that PEG was not present on the surface of the fibers. WCA 
measurements showed that the fiber mats were highly hydrophobic and surface rearrangement took place in an aqueous environment during the test. The tensile strength measured on fiber mat microdumbbells was comparable to that of soft human tissues. Cell culture studies demonstrated that the fiber mats were non-cytotoxic. Due to the excellent bioinertness and TPE properties, these fiber mats hold great promise as drug-eluting implant coatings. These drug-eluting fiber mats could be the solution to mitigate the capsular contracture around silicone rubber breast prostheses. The experiments for in vitro and in vivo drug release from AIBA/PEG fiber mats are in progress.

\section{Acknowledgements}

We would like to thank Dr. George Chase for FibraQuant software, Dr. Bi-min Zhang Newby for WCA measurements, and Dr. George Nikolov for XPS measurements, all at the University of Akron.

\section{Funding}

This material is based upon work supported by the National Science Foundation under Grant No. IIP-1434014 (Accelerating Innovation Research). Any opinions, findings, and conclusions or recommendations expressed in this material are those of the author(s) and do not necessarily reflect the views of the National Science Foundation. Kolos Molnáar would like to thank PolyFiberMatrix LLC and the Fellowship of the Hungarian-American Enterprise Scholarship Fund (HAESF) for financial support.

\section{REFERENCES}

[1] Doshi, J.; Reneker, D.H. Electrospinning Process and Applications of Electrospun Fibers. J. Electrostat. 1995, 35, 151-160. DOI: 10.1016/0304-3886(95)00041-8. 
[2] Choi, S.-S.; Hong, J.-P.; Seo, Y. S.; Chung, S. M.; Nah, C. Fabrication and Characterization of Electrospun Polybutadiene Fibers Crosslinked by UV Irradiation. J. Appl. Polym. 2006, 101, 2333-2337. DOI: 10.1002/app.23764.

[3] Duong, H.C., Chuai, D., Woo, Y.C., Shon, H.K., Nghiem, L.D. and Sencadas, V. A Novel Electrospun, Hydrophobic, and Elastomeric Styrene-Butadiene-Styrene Membrane for Membrane Distillation Applications. J. Membrane Sci. 2018, 549, 420-427. DOI: 10.1016/j.memsci.2017.12.024.

[4] Chuangchote, S., Sirivat, A., Supaphol, P. Electrospinning of Styrene-Isoprene Copolymeric Thermoplastic Elastomers. Polym. J. 2006, 38, 961-969. DOI: 10.1295/polymj.PJ2005234.

[5] Rungswang, W., Kotaki, M., Shimojima, T., Kimura, G., Sakurai, S., Chirachanchai, S. Existence of Microdomain Orientation in Thermoplastic Elastomer Through a Case Study of SEBS Electrospun Fibers. Polymer 2011, 52, 844-853. DOI: 10.1016/j.polymer.2010.12.019.

[6] Kamath, K.R., Barry, J.J., Miller, K.M. The Taxus ${ }^{\mathrm{TM}}$ Drug-eluting Stent: A New Paradigm in Controlled Drug Delivery, Adv. Drug. Deliver. Rev. 2006, 58, 412-436, DOI: 10.1016/j.addr.2006.01.023.

[7] Puskas, J.E., Dos Santos, L.M., Orlowski, E. Polyisobutylene-based Thermoplastic Biorubbers. Rubber Chem. Technol. 2010, 83, 235-246. DOI: 10.5254/1.3525683.

[8] Puskas, J.E., Kwon Y., Anthony P., Bhowmick, A.K. Synthesis and Characterization of Novel Dendritic (Arborescent, Hyperbranched) Polyisobutylene-Polystyrene Block Copolymers. J. Polym. Sci. A1. 2005, 43, 1811-1826. DOI: 10.1002/pola.20638. 
[9] Lim, G.T., Valente, S.A., Hart-Spicer, C.R., Evancho-Chapman, M.M., Puskas, J.E., Horne, W.I., Schmidt S.P. New Biomaterial as a Promising Alternative to Silicone Breast Implants, J. Mech. Behav. Biomed. Mater. 2013, 21, 47-56. DOI: 10.1016/j.jmbbm.2013.01.025.

[10] Puskas, J.E., Hoerr, R.A. Drug Release from Novel Rubbery Coatings. Macromol. Sy. 2010, 291-292, 326-329. DOI: 10.1002/masy.201050538.

[11] Liu, Y., Liu, X., Chen, J. Gilmore, K.J., Wallace G.G. 3D Bio-nanofibrous PPy/SIBS Mats as Platforms for Cell Culturing, Chem. Commun. 2008, 32 3729-3731. DOI: 10.1039/b804283g.

[12] Lim, G.T., Puskas, J.E., Reneker, D.H., Jakli, A., Horton, W.E. Highly Hydrophobic Electrospun Fiber Mats from Polyisobutylene-Based Thermoplastic Elastomers. Biomacromolecules. 2011, 12, 1795-1799, DOI: 10.1021/bm200157b.

[13] Jindal, A., Puskas, J.E., McClain, A., Nedic, K., Luebbers, M.T., Baker Jr, J.R., Dos Santos, B.P., Camassola M., Jennings, W., Einsporn, R.L., Leipzig, N.D. Encapsulation and Release of Zafirlukast from Electrospun Polyisobutylene-based Thermoplastic Elastomeric Fiber Mat, Eur. Polym. J. 2018, 98, 254-261, DOI: 10.1016/j.eurpolymj.2017.11.012.

[14] Kim, B.H., Park, M., Park, H.J., Lee, S.H., Choi, S.Y., Park, C.G., Han, S.M., Heo, C.Y., Choy, Y.B. Prolonged Acute Suppression of Cysteinyl Leukotriene to Reduce Capsular Contracture Around Silicone Implants, Acta Biomater. 2017, 51, 209-219. DOI: 10.1016/j.actbio.2017.01.033.

[15] Gergely, A.L., Puskas, J.E. Synthesis and Characterization of Thermoplastic Elastomers with Polyisobutylene and Polyalloocimene Blocks. J. Polym. Sci. A1. 2015, 53, 1567-1574. DOI: 10.1002/pola.27587. 
[16] Goldblatt, L.A., Palkin, S. Vapor Phase Thermal Isomerization of $\alpha$ - and $\beta$-Pinene. J. Am. Chem. Soc. 1941, 63, 3517-3522. DOI: 10.1021/ja01857a075.

[17] ISO/IEC 10993-6: Biological Evaluation of Medical Devices, Tests for In-vitro Cytotoxicity, 2009.

[18] Koombhongse, S., Liu, W., Reneker, D.H. Flat Polymer Ribbons and Other Shapes by Electrospinning. J. Polym. Sci. B. Polym. Phys. 2001, 39, 2598-2606. doi:10.1002/polb.10015.

[19] Albarran, A.A., Rosenthal-Kim, E.Q., Kantor, J., Liu, L., Nikolov, Z., Puskas, J.E. Stimuliresponsive Antifouling Polyisobutylene-based Biomaterials via Modular Surface Functionalization, J. Polym. Sci. A1, 2017, 55, 1742-1749. DOI: 10.1002/pola.28540.

[20] Puskas, J.E., Chen, Y. Biomedical Application of Commercial Polymers and Novel Polyisobutylene-based Thermoplastic Elastomers for Soft Tissue Replacement, Biomacromolecules 2004, 5, 1141-1154. DOI: 10.1021/bm034513k. 